
$51 / 1595850$

PREPARED FOR THE U.S. DEPARTMENT OF ENERGY, UNDER CONTRACT DE-AC02-76-CHO-3073

4. PPPL-3115

UC-426

ALFVÉN FREQUENCY MODES AT THE EDGE OF TFTR PLASMAS

BY

PPPL-3115

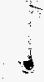

\author{
Z. CHANG, E.D. FREDRICKSON, S.J. ZWEBEN, ET AL.
}

\author{
JULY 1995
}
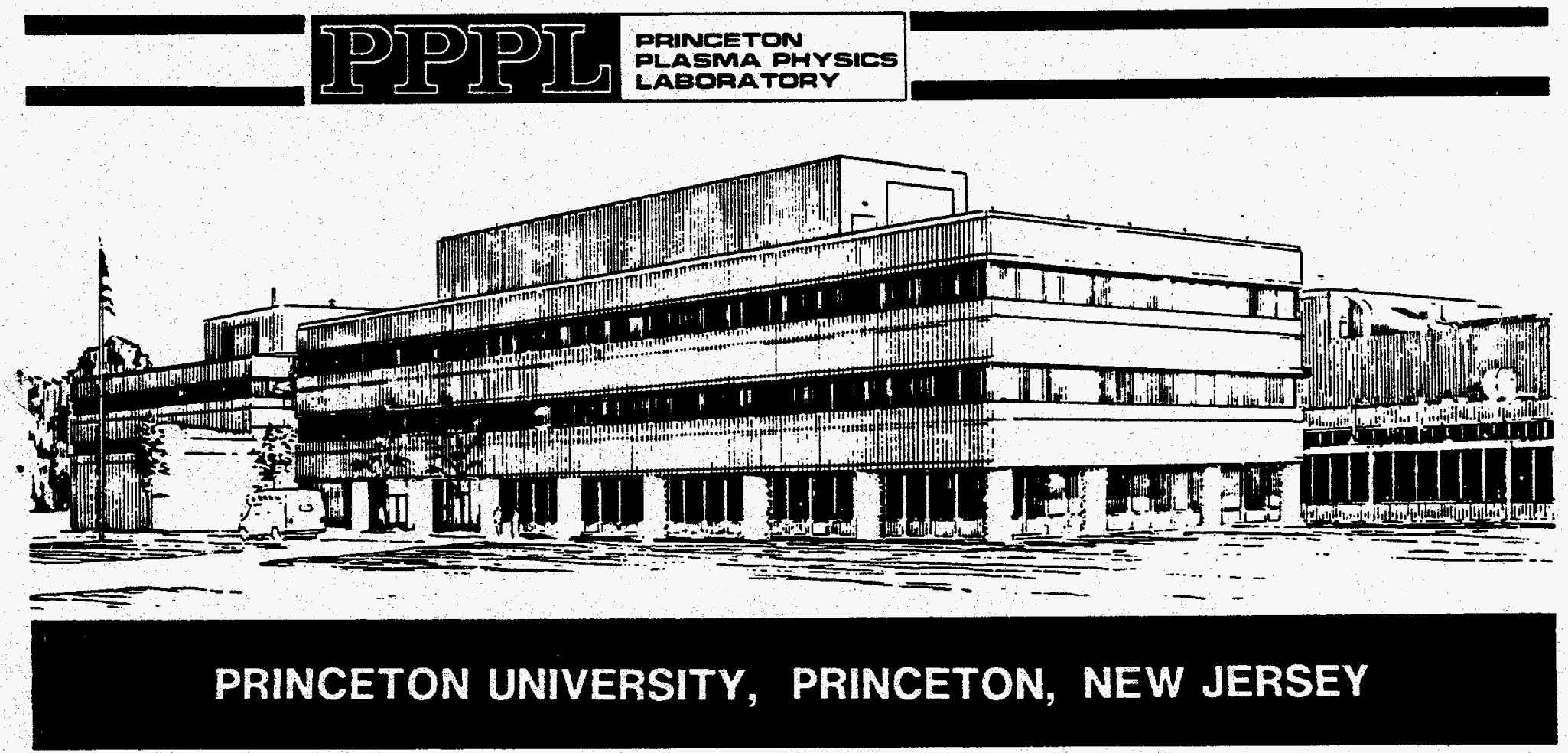


\section{NOTICE}

This report was prepared as an account of work sponsored by an agency of the United States Government. Neither the United States Government nor any agency thereof, nor any of their employees, makes any warranty, express or implied, or assumes any legal liability or responsibility for the accuracy, completeness, or usefulness of any information, apparatus, product, or process disclosed, or represents that its use would not infringe privately owned rights. Reference herein to any specific commercial produce, process, or service by trade name, trademark, manufacturer, or otherwise, does not necessarily constitute or imply its endorsement, recommendation, or favoring by the United States Government or any agency thereof. The views and opinions of authors expressed herein do not necessarily state or reflect those of the United States Government or any agency thereof.

\section{NOTICE}

This report has been reproduced from the best available copy. Available in paper copy and microfiche.

Number of pages in this report: 30

DOE and DOE contractors can obtain copies of this report from:

Office of Scientific and Technical Information

P.O. Box 62

Oak Ridge, TN 37831 ;

(615) $576-8401$.

This report is publicly available from the:

National Technical Information Service

Department of Commerce 5285 Port Royal Road

Springfield, Virginia 22161

(703) $487-4650$ 


\section{DISCLAIMER}

Portions of this document may be illegible in electronic image products. Images are produced from the best available original document. 


\title{
Alfvén Frequency Modes at the Edge of TFTR Plasmas
}

\author{
Z. Chang, E. D. Fredrickson, S. J. Zweben, H. Park, R. Nazikian, \\ E. Mazzucato, S. Batha ${ }^{\alpha}$, M. G. Bell, R. V. Budny, C. E. Bush, D. S. Dar- \\ row, D. Ernst ${ }^{b}$, G. Y. Fu, R. J. Hawryluk, K. W. Hill, J. Hosea, \\ A. C. Janos, D. L. Jassby, D. W. Johnson, L. C. Johnson, F. M. Levinton ${ }^{a}$, \\ D. K. Mansfield, K. M. McGuire, D. R. Mikkelsen, D. Mueller, D. K. Owens, \\ A. Ramsey, S. A. Sabbagh ${ }^{c}$, S. D. Scott, E. J. Synakowski, H. Taka- \\ hashi, G. Taylor, M. E. Thompson, R. M. Wieland, K. L. Wong and \\ M. C. Zarnstorff
}

Plasma Physics Laboratory, P. O. Box 451, Princeton University, Princeton, NJ 08543, USA,

${ }^{a}$ Fusion Physics and Technology, Torrance, CA

${ }^{b}$ Massachusetts Institute of Technology, Cambridge, MA

${ }^{c}$ Columbia University, New York, NY

\begin{abstract}
An Alfvén frequency mode (AFM) is very often seen in TFTR neutral beam heated plasmas as well as ohmic plasmas. This quasi-coherent mode is so far only seen on the magnetic fluctuation diagnostics (Mirnov coils). A close correlation between the plasma edge density and the mode activity (frequency and amplitude) has been observed, which indicates that the AFM is an edge localized mode with $r / a>0.85$. No direct impact of this mode on the plasma global performance or fast ion loss (e.g., the $\alpha$-particles in DT experiments) has been observed. This mode is apparently not the conventional TAE (toroidicity-induced Alfvén eigenmodes). The present TAE theory cannot explain the observation. Other possible explanations are discussed.
\end{abstract}

DISTRIBUTION OF THIS DOCUMENT IS UNLIMITED 


\section{INTRODUCTION}

A high frequency $(\sim 200-400 \mathrm{kHz})$ mode is often observed in the magnetic fluctuation measurement (Mirnov coils) in TFTR plasmas with neutral beam injection (NBI) heating. Since the frequency range is around the Toroidicity-induced Alfvén Eigenmodes (TAE)[1] frequency, this mode has been considered a candidate for the hot-ion driven TAE. Due to the potential importance of TAE modes in the fusion reactor devices, like ITER (International Thermonuclear Experimental Reactor), understanding of this mode becomes an important issue in the fusion research.

In recent high power DT operation in TFTR, a preliminary analysis showed that there is some enhancement of the mode amplitude in the high fusion power discharges[2]. This observation has led to a speculation that this mode may be the $\alpha$-driven TAE mode which corresponds to a theoretically predicted core localized $\alpha$-driven TAE mode[3]. We will show in this paper that this Alfven frequency mode (AFM) is not the conventional TAE mode. It is located near the plasma edge and has no direct impact on plasma global performance and has no observable effect on fusion or other energetic ions. On the other hand, due to its location the AFM has a strong correlation with the plasma-wall interaction.

In Section 2 we will first summarize the mode characteristics. Section 3 shows how the mode location is determined from the correlation between the changes observed in the mode frequency and electron density measurements. A scaling analysis of the AFM amplitude given in Section 4 exhibits some interesting plasma parameter dependence.

The most striking observation is the existence of the AFM in ohmic plasmas. The ohmic-AFM discussed in Section 5 is similar to the ones observed in NB plașmas. This observation clearly indicates that the excitation of the AFM does not rely on the presence of an energetic ion species. In Section 6 we briefly discuss the theoretical issues raised from the usual TAE theory and introduce another possible candidate related to the edge charge-exchange process. Finally the conclusions are given in Section 7. 


\section{Characteristics of the Alfvén-Frequency-Mode}

The basic characteristics of the AFM have been briefly reported recently[4]. A typical example in a deuterium NBI-heated plasma in TFTR is shown in Fig. 1. The mode usually starts $\sim 50-100 \mathrm{~ms}$ after the beam injection and the amplitude slowly increases. It takes about 200-300 $\mathrm{ms}$ for the mode to saturate. The decay time of the mode in the post neutral beam phase is even longer, typically $\geq 0.5$ sec. At present, Mirnov coils[5] are the only diagnostic system in TFTR that can observe the mode.

An important feature of the Alfvén frequency mode is the quasi-coherency. Figure 2 shows the frequency spectrum of the mode compared with the TAE modes driven by the energetic tail ions in the ICRF-heated plasmas[6]. The frequency band width of the AFM is typically about $50 \mathrm{kHz}$, while a single $n$ (the toroidal mode number) TAE mode has a width $\lesssim 1 \mathrm{kHz}$. The AFM often consists of two bumps. The second higher frequency peak is usually weaker and looks narrower in frequency. However, it can be greatly enhanced in the current ramp-down $\mathrm{H}$-mode case. The two AFM bumps can reach to the same amplitude. In some circumstances, the higher frequency bump can become larger than the lower frequency bump.

The measured mode number for the AFM is also very different from the TAE modes as shown in Fig. 2. The phase shift measurement from the Mirnov coil array gives $n=0$ for both bumps, i.e., the AFM is an axisymmetric mode. A standing wave structure is often seen in the phase shift analysis of the poloidal coil array. The poloidal number $m$ is typically 1 or 2 . The poloidal variation of the AFM is also very different from the RF-driven TAE mode. As we can see from Fig. 3 that the TAE mode has a "ballooning" feature, namely its amplitude peaks on the lower $B$ field side. On the other hand the AFM exhibits a "anti-ballooning" feature (the plasma is contacting the inner bumper limiter).

Among various coherent MHD modes observed in TFTR, the AFM is the weakest. Typically, the measured edge magnetic fluctuation level is $\sim \mathcal{O}\left(10^{-4}\right)$ Gauss, and

$$
\begin{aligned}
& \tilde{B}_{\theta}^{A F M} / B_{0} \lesssim 10^{-7}, \\
& \tilde{B}_{\theta}^{A F M} / \tilde{B}_{\theta}^{f i s h b o n e} \lesssim 10^{-3},
\end{aligned}
$$




$$
\begin{aligned}
& \tilde{B}_{\theta}^{A F M} / \tilde{B}_{\theta}^{\text {tearing }} \leqslant 10^{-2}, \\
& \tilde{B}_{\theta}^{A F M} / \tilde{B}_{\theta}^{T A E} \leqslant 10^{-1} .
\end{aligned}
$$

Despite its weakness, the AFM is the most common MHD activity observed in routine TFTR discharges. It has been seen in almost all of the operational regimes, e.g., L-mode, supershot[7], high- $\beta_{p}[8]$, limiter H-mode[9], and even in some ohmic plasmas.

\section{Determination of the AFM location}

A very striking feature of the AFM is its strong correlation with edge density influx events, for example, gas puff, pellet injection, impurity "bloom", strong wall de-conditioning induced by minor disruption or $\beta$ collapse, etc. It is this feature that allows us to determine the mode location.

A good example of this correlation in a DT experiment is shown in Fig. 4, where a Helium gas puff is introduced during the NBI phase. Following the puff, the AFM frequency decreases and the amplitude increases. On TFTR, line integral electron density is measured by multichannel infrared interferometer (MIRI) system[10]. The spatial and temporal electron density information is obtained via an accurate Abel inversion method[11] supported by a practical error analysis[12] of this method. The correlation between the changes of the AFM frequency and the electron density at different radii is illustrated in Fig. 4. The $n_{e}$ evolution shown at Fig. 4(b) clearly exhibits a propagation of the incremental $n_{e}$ from the edge to the center after the puff. A sharp drop of the $f_{A F M}$ closely follows the gas puff, or the $n_{e}$ near the edge $(R>300 \mathrm{~cm})$. In addition, a small $n_{e}$ burst event can be seen at the edge channels $(R \gtrsim 335 \mathrm{~cm}$ and $R \lesssim 180 \mathrm{~cm})$ at $3.74 \mathrm{sec}$. A corresponding drop in $f_{A F M}$ is also clearly seen in the AFM frequency spectrum contour plot Fig. 4(c).

In order to show that this mode is an Alfvén mode, we plot the edge Alfvén frequency in Fig. 4(c). Here, the $f_{\text {Alfvén }}$ is defined as

$$
\begin{aligned}
f_{\text {Alfvén }} & \equiv \frac{V_{A}}{2 \pi q R} \\
& =3.47 \times 10^{11} \frac{B_{0}(T)}{q R_{0}(\mathrm{~cm}) \sqrt{\sum_{i} A_{i} n_{i}\left(\mathrm{~cm}^{-3}\right)}} \quad(\mathrm{kHz})
\end{aligned}
$$


where $V_{A}=B / \sqrt{4 \pi \sum_{i} n_{i} m_{i}}$ is the Alfvén velocity. For simplicity, we use the magnetic field and major radius at the center of the outer most plasma flux surface denoted as $B_{0}$ and $R_{0}$. The $A_{i}$ is the mass ratio of the ion to the proton. The $q(r, t)$ and $n_{i}(r, t)$ are from the transport simulation code TRANSP[13]. Based on the TFTR diagnostic measurements, we use

$$
\Sigma A_{i} n_{i} \simeq 2 n_{D}+3 n_{T}+2.5 n_{b}+n_{H}+4 n_{H e}+12 n_{I m p}
$$

where $n_{D}, n_{T}, n_{b}, n_{H}, n_{H e}$ and $n_{I m p}$ are deuterium, tritium, beam(50-50 D-T mixture), hydrogen, helium and impurity(mainly carbon) ion density, respectively. Figure 4 (c) shows that within the measurement and simulation uncertainties (typically $\leqslant 10-20 \%$ ), the $f_{A F M}$ agrees well with the edge Alfven frequency. Since $n_{e}$ is a directly measured plasma quantity and has a higher time resolution (up to 0.5 $m s$ ) than the TRANSP simulation ( $\sim 10 \mathrm{~ms}$ ), we will use $2 n_{e}$ to replace $\Sigma A_{i} n_{i}$ in the Alfvén frequency calculation in the rest of paper. It is denoted as $f_{A l f}^{e}$ :

$$
f_{A l f}^{e} \equiv 3.47 \times 10^{11} \frac{B_{0}(T)}{q R_{0}(\mathrm{~cm}) \sqrt{2 n_{e}\left(\mathrm{~cm}^{-3}\right)}} \quad(\mathrm{kHz})
$$

It is found that the agreement between $f_{\text {Alfuen }}$ and $f_{A l f}^{e}$ is within $\leqslant 5 \%$. Note that since $\omega_{T A E} \simeq \omega_{A} / 2$, if the same formula as Eq. (2) is used to calculate the TAE frequency, there will be a factor of 2 difference between the edge $f_{T A E}$ and the measured AFM frequency. The same technique (correlation between the mode frequency and plasma density) has also been used to determine the location of a RF-driven TAE mode. It is found that the mode location estimated using this technique agrees well with the multi-channel Reflectometer measurement.

Recently, a numerical simulation shows that a core-localized $\alpha$-driven TAE mode may be unstable in a high fusion power discharge 76770[3]. The AFM activity in this discharge reveals the same characteristics. As shown in Fig. 5, near the end of the NBI phase, there is a small wall de-conditioning event indicated by an increase in the edge electron density, Fig. 5(a). The central density remains unperturbed. The calculated Alfvén frequencies normalized at $4.2 \mathrm{sec}$ using the edge and core $n_{e}$ again demonstrate that the AFM is not the core localized mode. The good agreement of the $f_{A F M}$ and the edge Alfven frequency indicates that the mode is localized at least outside $r / a>0.83$. The analysis of the Alfvén 
frequency mode shows no correlation between the observed AFM and the core TAE mode. The same phenomenon, i.e., an increase in the AFM amplitude and decrease in $f_{A F M}$ that is well correlated with the edge $n_{e}$, can also be seen in a pellet injection case as shown in Fig. 6 . Here, a much abrupt increase in $n_{e}$ induces a sharp drop in the $f_{A F M}$ and strong enhancement in $\dot{B}_{A F M}$.

A detailed comparison of different MHD activities is shown in Fig. 7. - As we can see that the internal MHD (sawtooth crash and fishbone activity) has no effect on the AFM. But the ELMs (edge localized modes) strongly affect the mode. Each ELM burst (see the $H_{\alpha}$ ) correlates to an AFM burst. Also, the AFM frequency shows a sharp drop at each ELM. Since the ELMs are originated from the high field side edge (near the inner bumper limiter), the good agreement between the $f_{A F M}$ and the Alfvén frequency at $R \simeq 174 \mathrm{~cm}$ Fig. 7(e) suggests that the AFM (at least the lower frequency branch) may be excited around the plasma-wall contacting region. In this case it is $\leqslant 8 \mathrm{~cm}$ from the inner bumper limiter $(R=165.6$ $\mathrm{cm})$. The higher frequency AFM branch exhibits the same behavior as the lower frequency main branch. But its frequency response to the edge $n_{e}$ perturbation is not as strong as the main branch. Since the Alfven frequency is a decrease function of plasma radius, we speculate that this mode may be located more inside than the main branch. It is found that a distance of $\sim 8 \mathrm{~cm}$ between the two modes is sufficient to account for the observed frequency difference.

The edge localization of the AFM mode can also explain the observation that no internal MHD modes have any strong interaction with the Alfvén frequency modes including the $q=1$ core MHD modes (sawtooth and fishbone), other internal MHD events happened in the confinement region $(r / a \sim 0.3-0.6)$, like the neoclassical tearing modes[14], the high frequency $(f \sim 50-200 \mathrm{kHz}$ ) KBM-like (kinetic ballooning mode) MHD[15] and the RF-driven TAE mode. Also, it can explain that the AFM has no direct impact on plasma global confinement and fast-ion (including $\alpha$-particle) losses.

\section{The AFM amplitude scaling}

To study the AFM instability characteristics we select a subset of the TFTR NBI-heated discharges. This database has more than 170 shots. It covers the major TFTR operational ranges: major radius $R_{0} \simeq 2.45,2.52$ and $2.60 \mathrm{~m}$; 
plasma current $I_{p} \simeq 1.0,1.2,1.4$ and 1.6 MA; neutral beam power $P_{B}=3-24$

MW. It also includes DD, DT and TT (T-beam only) discharges. The data are taken around $0.6 \mathrm{sec}$ after the start of beam injection. Large catastrophic events (disruption, impurity bloom, etc) are excluded.

First of all, we find that the AFM frequency scales well with the Alfvén frequency near the edge as shown in Fig. 8. Here, for simplicity we use the edge $q$ and $n_{e}$ at $R=180 \mathrm{~cm}$ (15cm from the inner limiter) for all the discharges. Figures $9(\mathrm{a})$ - $(\mathrm{c})$ show the mode amplitude $\left(\dot{B}_{A F M}\right)$ as a function of the total beam power $P_{B}$, total stored energy $E_{t o t}$, and the ratio of the co-direction (plasma current direction) beam power $P_{c o}$ and $P_{B}$. The statistical analysis shows that: (1) The AFMs are not enhanced in DT discharges. (2) There is no systematic dependence between the AFM amplitude and the beam power or plasma global performance (e.g., stored energy or confinement time). (3) The AFM does not depend on the plasma rotation (inferred by the ratio of $P_{c o} / P_{B}$ ). On the other hand, a strong correlation between the AFM amplitude and the edge density for a given current is observed, see Fig. 10(a), which agrees with the spontaneous correlation we discussed in Section 3. Figure 10(b) shows that for given $B_{0}$ and $R_{0}$, the saturated AFM amplitude $\left(\propto \dot{B}_{\theta}^{A F M} / f_{A F M}\right)$ scales roughly as

$$
\tilde{B}_{\theta}^{A F M} \propto q_{a}^{3} n_{e}^{1.5}
$$

This scaling is also confirmed by the time evolution of the AFM in a single discharge.

\section{The AFM in ohmic plasmas}

The most surprising observation is probably the AFM in the ohmic plasmas. In normal TFTR operation configuration with plasmas contacting the inner bumper limiter, the Alfvén frequency mode can be easily observed in ohmic plasmas after a pellet injection. The mode behaves very similar to the NB plasma case shown in Fig. 6. The pellet-induced ohmic-AFM also has two peaks. The higher frequency branch can be very coherent and lasts longer ( $\approx 0.5 \mathrm{sec}$ ) than the lower frequency main branch. Just after the pellet injection, the main branch can also become very coherent. Then, the peak quickly broadens. The mode also has a standing wave structure with $n=0$ and $m=1$. The basic feature does not 
depend on the pellet material (Lithium or Boron).

Since the magnetic coils designied to measure the toroidal wavelength of high frequency (for $f>250 \mathrm{kHz}$ ) fluctuations are located at the outboard side $\left(\sim 65^{\circ}\right.$ bellow the midplane), one would expect that the measurement of the AFM in a large major radius plasma with the plasma contacting to the outer RF-limiter will be more sensitive. Indeed, a clear AFM is observed in the entire pulse of an ohmic discharge in such a configuration. As shown in Fig. 11, the frequency of the ohmicAFM agrees very well with the edge (close to the RF-limiter) Alfvén frequency. Note that the plasma parameters change drastically in the whole discharge. The maximum mode amplitude is now peaked on the high $B$ field side, which agrees with the assumption that the mode originates around the plasma-wall contacting region.

\section{Discussion of the theoretical modeling}

The physical understanding of the edge AFM phenomenon is a challenging task. The conventional TAE theory encounters the following two major difficulties in explaining the AFM. First of all, the AFM frequency is usually in the Alfven continuum region. Figure 12 shows the calculated TAE gap structure (for $n=$ 3) for a DT discharge. It is from the NOVA-K code[16] calculation based on measured and TRANSP interpreted plasma parameters. The expected $\alpha$-driven TAE frequency is more than $40 \%$ lower than the AFM frequency. The lower frequency AFM branch is located inside the upper continuum of the first TAE gap. The higher frequency branch may be sitting in the second gap. Despite the different $n$ number used in the calculation (note that the AFM has $n=0$ ), the fact that the mode is inside the Alfven continuum means that the TAE instability should be prohibited.

Considering the many uncertainties possibly involved in both the measurement and numerical simulation, we may not exclude the possibility that the AFM could be located in one of the TAE gaps. However, since the conventional TAE instability theory relies on the resonance between the energetic particles and the MHD wave, the immediate question becomes: can we find such a driving source at the plasma edge? Detailed calculation using the NOVA-K code shows that even the TAE modes which usually locate around $r / a \sim 0.6-0.8$, are stable[17]. Namely, 
the present TFTR DD and DT discharges do not have sufficient drive for the TAE instability[3]. Therefore, near the plasma edge the wave-particle resonance is very unlikely playing any role in the AFM "instability". To have the ion velocity resonate with the edge Alfvén velocity, i.e., $v_{i} \sim V_{A}^{e d g e}$, the demand ion energy would be $E_{i}>1 \mathrm{MeV}$. In the ohmic plasmas one can hardly find such a fast-ion species. Also, the quasi-coherency of the mode seems to suggest that the mode is not driven by the wave-particle resonance.

To understand the AFM physics we may need to search for non-resonant "driving" mechanisms. It is well-known that Alfvén wave is a natural and damped mode in magnetically confined plasmas. Namely, if no sources exist, an Alfvén mode (from either external perturbation or intrinsic turbulent "noise") will be damped by various dissipation processes. This is why we don't see Alfvén modes in plasma interior without a sufficient drive. A speculation on the AFM phenomenon we observed is that the edge physics, e.g., neutral-ion charge exchange, ion/electron impeding ionization, radiation, ion-orbit loss, etc., may play a role to either reduce the dissipative damping or to enhance the thermal Alfvén "noise". To illustrate this thought process, we introduce a non-ideal MHD model including a charge-exchange term. We assume that the neutral particles coming from the wall carry certain non-isotropic velocity. Through charge-exchange (the dominant atomic process in tokamak edge) the neutral particles introduce a perturbation in the MHD momentum equation near the plasma edge (and few ion Larmor radii away from the wall to avoid the complication of the ion-orbit loss). The one-fluid MHD equation can be written as

$$
\begin{aligned}
n_{i} m_{i} \frac{\partial \mathbf{v}}{\partial t} & =(\nabla \times \mathbf{B}) \times \mathbf{B}-\nabla p+\nu \nabla^{2} \mathbf{v}+n_{i} m_{i} k_{c} n_{n} \mathbf{v} \\
\frac{\partial \mathbf{B}}{\partial t} & =\nabla \times(\mathbf{v} \times \mathbf{B})+\frac{\eta}{\mu_{0}} \nabla^{2} \mathbf{B}
\end{aligned}
$$

The last term in Eq. (6) is a model of the neutral-ion charge exchange contribution, where $k_{c}$ can be considered an averaged charge exchange rate, $n_{n}$ is the neutral density. The dissipation processes are parameterized by the viscosity $\nu$ and resistivity $\eta$. In a slab geometry we denote that $x$ is in the plasma gradient direction, $z$ is parallel to the $\mathbf{B}_{0}$ and $y$ is in the transverse direction (corresponding to the poloidal direction in a torus). A dispersion equation can be easily derived from 
Eqs. (6) and (7):

$$
\left[\left(\omega-i k_{c} n_{n}+i \frac{\nu}{n_{i} m_{i}} k_{\perp}^{2}\right)\left(\omega+i \frac{\eta}{\mu_{0}} k_{\perp}^{2}\right)-k_{\|}^{2} V_{A}^{2}\right] \tilde{v}_{y}=0 .
$$

Assuming a weak growth rate $\left(\gamma \ll \omega_{r}\right)$, we obtain the Alfvén mode instability:

$$
\begin{gathered}
\omega_{r}^{2}=k_{\|}^{2} V_{A}^{2}, \quad \omega_{r}= \pm k_{\|} V_{A} \\
\gamma=\frac{1}{2}\left[k_{c} n_{n}-\left(\frac{\nu}{n_{i} m_{i}}+\frac{\eta}{\mu_{0}}\right) k_{\perp}^{2}\right] .
\end{gathered}
$$

Equation (9) indicates that the Alfvénic wave propagates in both directions. Therefore, it has a standing wave phase structure as we observed in the experiments. In Eq. (10), the second term is the usual dissipative damping term. The first driving term is from the charge-exchange contribution. For typical TFTR plasma edge parameters, we found that the driving term and the damping term are in the same order. Namely,

$$
\begin{aligned}
& k_{c} n_{n} \sim 10^{-8} \mathrm{~cm}^{3} / \mathrm{sec} \times 10^{9} \mathrm{~cm}^{-3} \sim \mathcal{O}(10) \quad 1 / \mathrm{sec}, \\
& \frac{\eta}{\mu_{0}} k_{\perp}^{2} \sim 10^{4} \mathrm{~cm}^{2} / \mathrm{sec} \times 10^{-3} \mathrm{~cm}^{-2} \sim \mathcal{O}(10) \quad 1 / \mathrm{sec} .
\end{aligned}
$$

This model has the following interesting features: (1) Driving mechanism is "thermal" or non-resonant. (2) Increasing in the edge density and edge ion temperature $\left(k_{c} \propto T_{i}^{0.3}\right.$, see for instance [[18]]), or decreasing in edge dissipation can excite the mode. (3) Long growth/decay time, $\gamma^{-1} \sim 100 \mathrm{~ms}$. These features seem to be not inconsistent with the observations. It is worth to note that in the well-conditioned supershot plasmas (e.g., using intensive Lithium pellet injection[19]) the AFM is found to be strongly suppressed. Since those plasmas have lower edge recycling rate and small edge density, weak AFM is expected from Eq. (10). Obviously, more work needs to be done in order to better understand the physics of the AFM phenomena.

\section{Conclusions}

The Alfvén frequency mode observed in the magnetic frequency spectrum is the most common MHD activity in TFTR routine operation. Observation of the close correlation between the changes in the AFM frequency and the local 
plasma density allows us to locate the mode and therefore, to understand and explore many interesting physics related to this mode. Through detailed analysis we showed that the AFM is an edge localized mode with $r / a>0.85$. It is very likely originated around the plasma-wall contacting region. There are many differences between the AFM and TAE modes. The AFMs are quasi-coherent and have a standing wave structure with $n=0$. Statistical data analysis shows that there is no correlation between the AFM and plasma global performance. The mode amplitude has a strong scaling with edge $q$ and a more-than-linear scaling on edge $n_{e}$. Observation of the ohmic-AFM indicates that the existence of the mode does not depend on the energetic particles. There is no satisfactory theory to explain the AFM phenomenon. A preliminary model based on the charge-exchange process suggests the possible importance of the edge physics. Although the mode has no direct impact on the plasma global performance, how the mode influences the plasma-wall interaction and plasma recycling is still a remaining area to be investigated in the future.

\section{ACKNOWLEDGMENTS}

The authors would like to thank Dr. J. Strachan, Dr. S. Paul, Dr. L. Chen, Dr. C. Z. Cheng, Dr. D. Spong, Dr. J. D. Callen and Dr. R. White for useful discussions. This research was supported by US DoE Contract No. DE-AC02-76CHO-3073. 


\section{References}

[1] CHENG, C. Z. and CHANCE, M. S., Phys. Fluids 29, (1986) 3695.

[2] FREDRICKSON, E. D., et al., in Proceedings of the 15th Conference on Plasma Physics and Controlled Nuclear Fusion Research (Seville, Spain, 1994), to be published by IAEA, Vienna, 1995. MEADE, D. M. and the TFTR Team, Journal of Fusion Energy 13, Nos. 2/3, 145 (1994). McGUIRE, K. M., et al., 36th APS review paper to be published in Phys. of Plasmas special issue, 1995.

[3] CHENG, C. Z., et al., in Proceedings of the 15th Conference on Plasma Physics and Controlled Nuclear Fusion Research (Seville, Spain, 1994), to be published by IAEA, Vienna, 1995. BERK, H. L. and VAN DAM, J. W., "More on Core-Localized Toroidal Alfvén Eigenmodes", Report IFSR\#695, 1995.

[4] FREDRICKSON, E. D., et al., in Proceeding of the 21st EPS Conference on Controlled Fusion and Plasma Physics, Vol. 18B Part I p. 246, 1994.

[5] FREDRICKSON, E., et al., Rev. Sci. Instrum. 57 (1986) 2084.

[6] WONG, K. L., et al., Plasma Phys. Controlled Fusion 36 (1994) 879.

[7] STRACHAN, J. D., et al., Phys. Rev. Lett. 58 (1987) 1004.

[8] SABBAGH, S. A., et al., in Proceedings of the 15th Conference on Plasma Physics and Controlled Nuclear Fusion Research (Seville, Spain, 1994), to be published by IAEA, Vienna, 1995.

[9] BUSH C., et al., in Proceedings of the 15th Conference on Plasma Physics and Controlled Nuclear Fusion Research (Seville, Spain, 1994), to be published by IAEA, Vienna, 1995.

[10] MANSFIELD, D. K., et al., Appl. Opt. 26 (1987) 4469.

[11] PARK, H. K.,Plasma Phys. Controlled Fusion 31 (1989) 2035.

[12] PARK, H. K., Rev. Sci. Instrum. 61 (1990) 2879. 
[13] BUDNY, R. V., Nucl. Fusion 34, 1247 (1994) and references there in.

[14] CHANG, Z., et al., "Observation of Nonlinear Neoclassical $\nabla p$-Driven Tearing Modes in TFTR", report PPPL-2988, 1994, to be published in Phys. Rev. Lett. .

[15] CHANG, Z., et al., Bull. of American Phys. Society, 39 (1994) 1697. NAZIKIAN, R., et al., Bull. of American Phys. Society, 39 (1994) 1672.

[16] CHENG, C. Z., Phys. Reports, 12111 (1992).

[17] ZWEBEN, S., private communication.

[18] NEDOSPASOV, A. V. and TOKAR', M. Z., Reviews of Plasma Physics, Vol 18 , p. 90.

[19] MANSFIELD, D. K., et al., "Enhanced D-T Supershot Performance at High Current Using Extensive Lithium Conditioning in TFTR", report PPPL3097, 1995, submitted to Phys. Plašmas. 


\section{List of Figures}

1 The Alfvén frequency mode is often seen on the magnetic fluctuation spectrum measured by Mirnov coils in NB-heated plasmas in TFTR. The mode amplitude is usually much weaker than other coherent

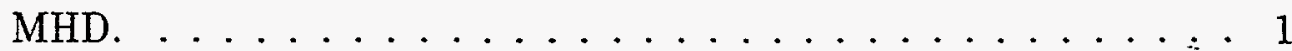

2 Comparison of the mode coherency between (a) the AFM and (b) the RF-driven TAE. Both AFM peaks are quasi-coherent with toroidal mode number $n=0 . \ldots \ldots \ldots 17$

3 Comparison of the poloidal variation of the mode amplitude for (a) the AFM and (b) the RF-driven TAE. Here, $0^{\circ}$ is at the outer midplane. Positive angle corresponds to the upper part. The noise level is also shown. A "ballooning" feature is clearly seen in the TAE case while the AFM shows an anti-ballooning feature. . . . .

4 (a) Profile evolution of the electron density after a gas puff in a DT plasma. The location of the Multichannel Infrared Interferometer (MIRI) is shown on the top. The time evolution of $n_{e}$ in different radii is shown in (b). (c) shows the contour plot of the magnetic fluctuation spectrum. A good correlation can be seen between the changes of the edge $n_{e}$ [also denoted in the shaded region in (a)] and the AFM frequency. The TRANSP calculated local Alfven frequency at $R=330 \mathrm{~cm}$ is shown in (c) . . . . . . . . . 19

5 The AFM in the high fusion power discharge (76770). (a) A wall de-conditioning event near the end of NB phase can be seen from the edge density evolution. (b) This event causes a decrease in $f_{A F M}$ and an increase in the mode amplitude. Comparison of the core and edge Alfvén frequencies with the measurement shows that the AFM is not a core mode. . . . . . . . . . . . . . . . . . .

6 The AFM in a pellet injection case (during the NB phase). (a) Density evolution at three different radii. (b) The mode frequency evolution agrees well with the Alfvén frequency using the density at $r / a=0.9$. (c) The AFM amplitude is strongly enhanced after the pellet injection. ................... 2 
7 Detailed correlation between the AFM and other MHD shows that the AFM activity $[(\mathrm{g})$ and $(\mathrm{h})]$ has no correlation to the sawtooth and fishbone MHD [(a) and (b)], but has strong correlation with the ELMs (f). The density perturbation at different plasma radii [(c)(e)] suggests that the AFM is originated around the inner bumper limiter since only the Alfvén frequency derived from the inside channel (e) can match the changes in the $f_{A F M}$, see $(\mathrm{h}) \ldots \ldots 22$

8 Statistical data analysis based on more than 170 neutral beam discharges. The AFM frequency is proportional to the Alfven frequency. Here, $f_{A l f}$ is calculated using the edge $q$ and $n_{e}$ at $R \simeq 180$ $\mathrm{cm}$

9 No systematic correlation has been found between the AFM amplitude and DD/DT variation, neutral beam power (a), total stored energy (b) and plasma rotation inferred by the ratio of the co-beam power versus total beam power (c). . . . . . . . . . . 24

10 (a) The AFM amplitude depends on the edge density. The correlation is stronger for lower plasma current. (b) For given $R_{0}$ and $B_{t}$, the mode amplitude scales roughly as $q^{3} n_{e}^{1.5} \ldots \ldots \ldots 2$

11 The AFM in an ohmic plasma. (a) Toroidal magnetic field. (b) Plasma major radius. The plasma is contacting the outer RFlimiter. (c) Edge $q$. (d) Edge electron density at $R=352 \mathrm{~cm}$ or $\sim 7 \mathrm{~cm}$ inside the RF-limiter. (e) Contour plot of the magnetic fluctuation spectrum. The AFM is clearly seen throughout the entire discharge. Due to memory limitation, the data is taken on three discrete blocks. The calculated Alfvén frequency using the parameters from (a)-(d) is also shown on (e). Some early bursts shown in (e) correlate to the external kink MHD activities. The higher frequency AFM branch can also be seen near the end of the discharge. 26

12 The TAE gap structure calculated from the NOVA-K code for $n=3$. The observed AFM frequency (the two branches shown in shaded region) is in the continuum. The simulation shows that the plasma should be stable to the $\alpha$-driven TAE. 


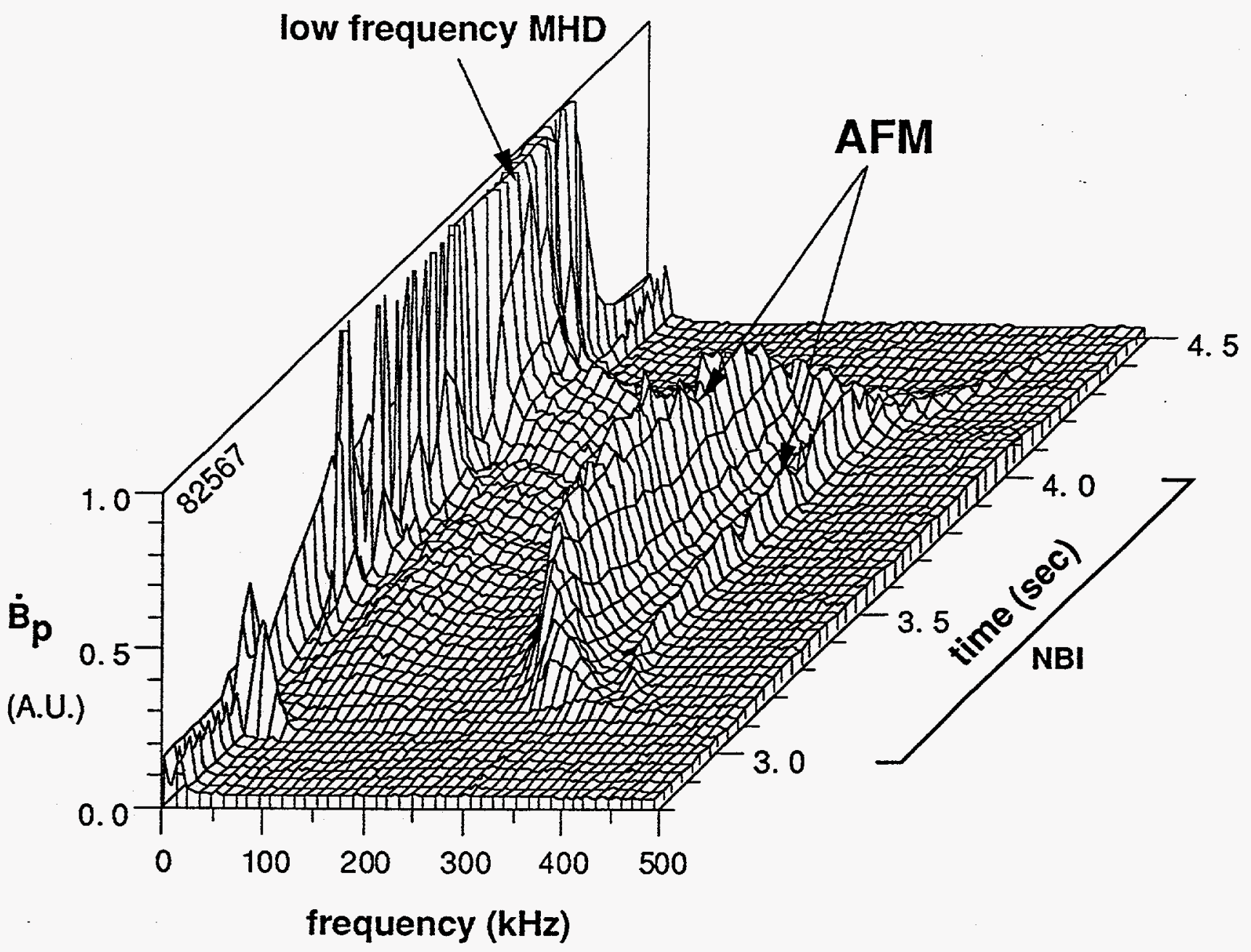

Fig. 1 


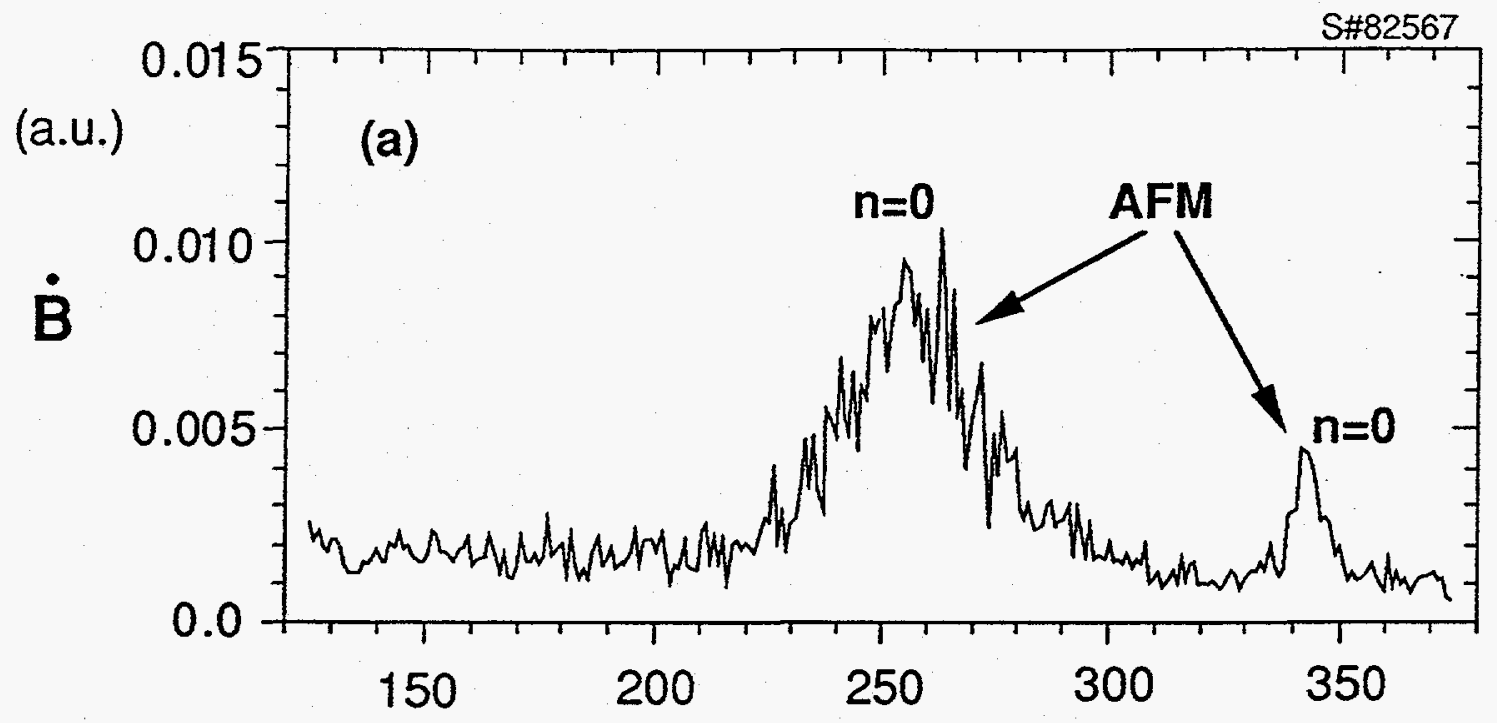

(a.u.)

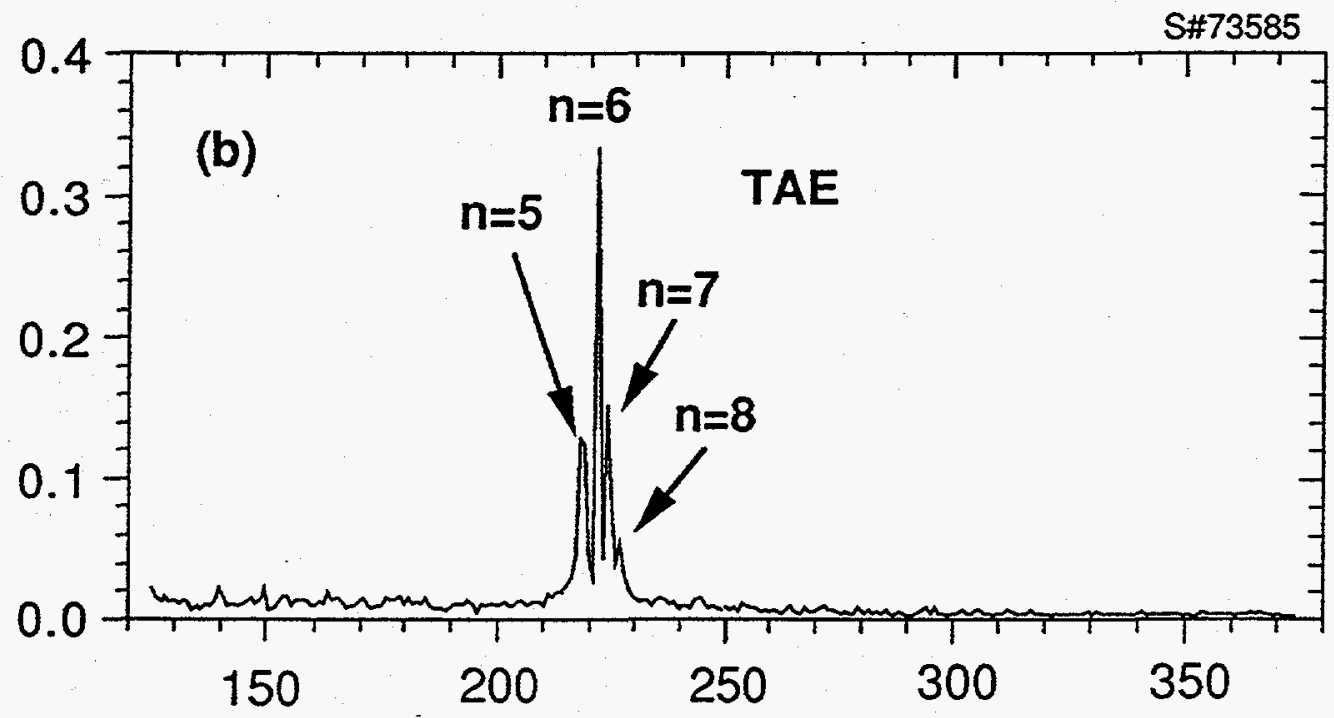

frequency $(\mathrm{kHz})$

Fig. 2 

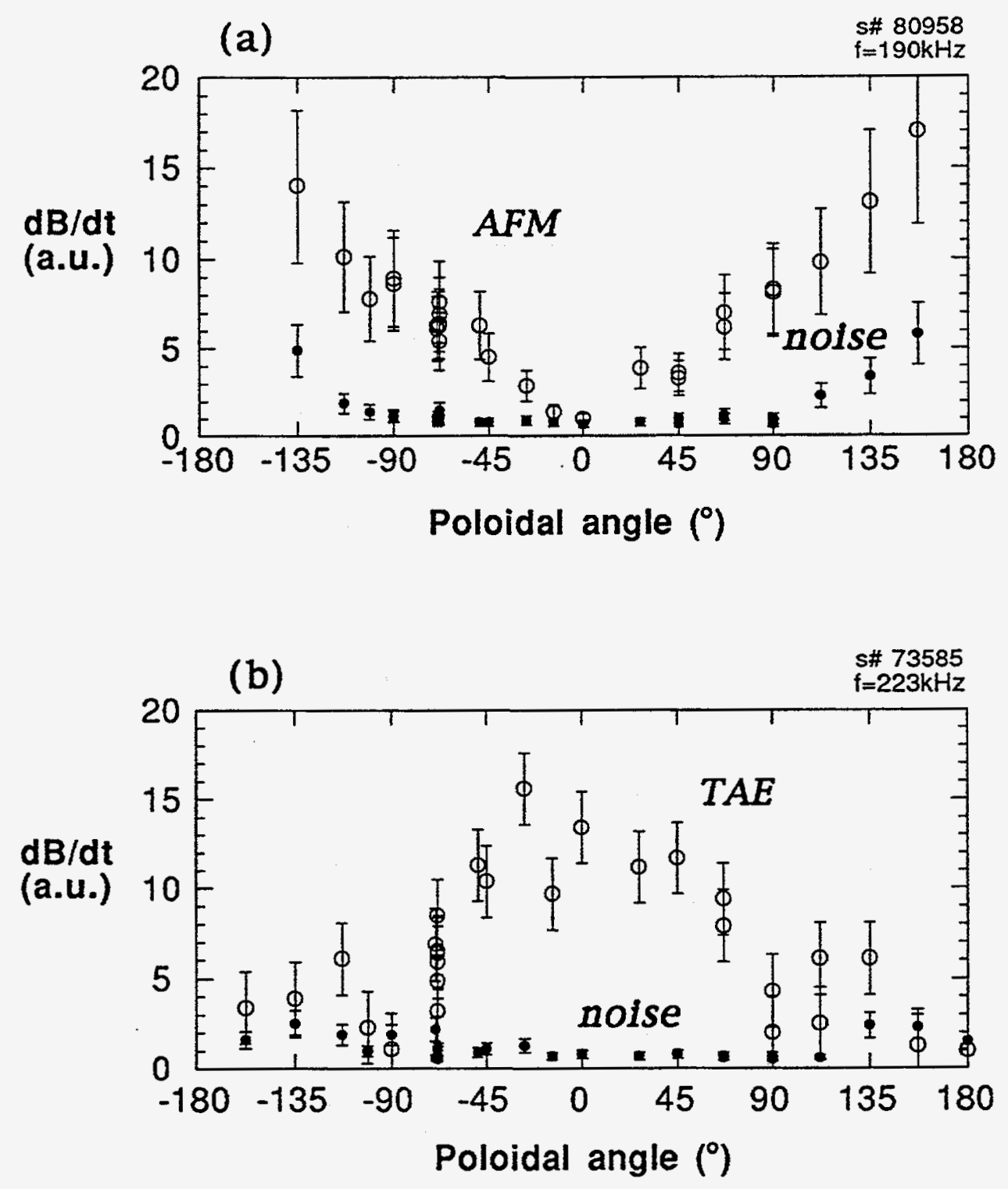

Fig. 3 


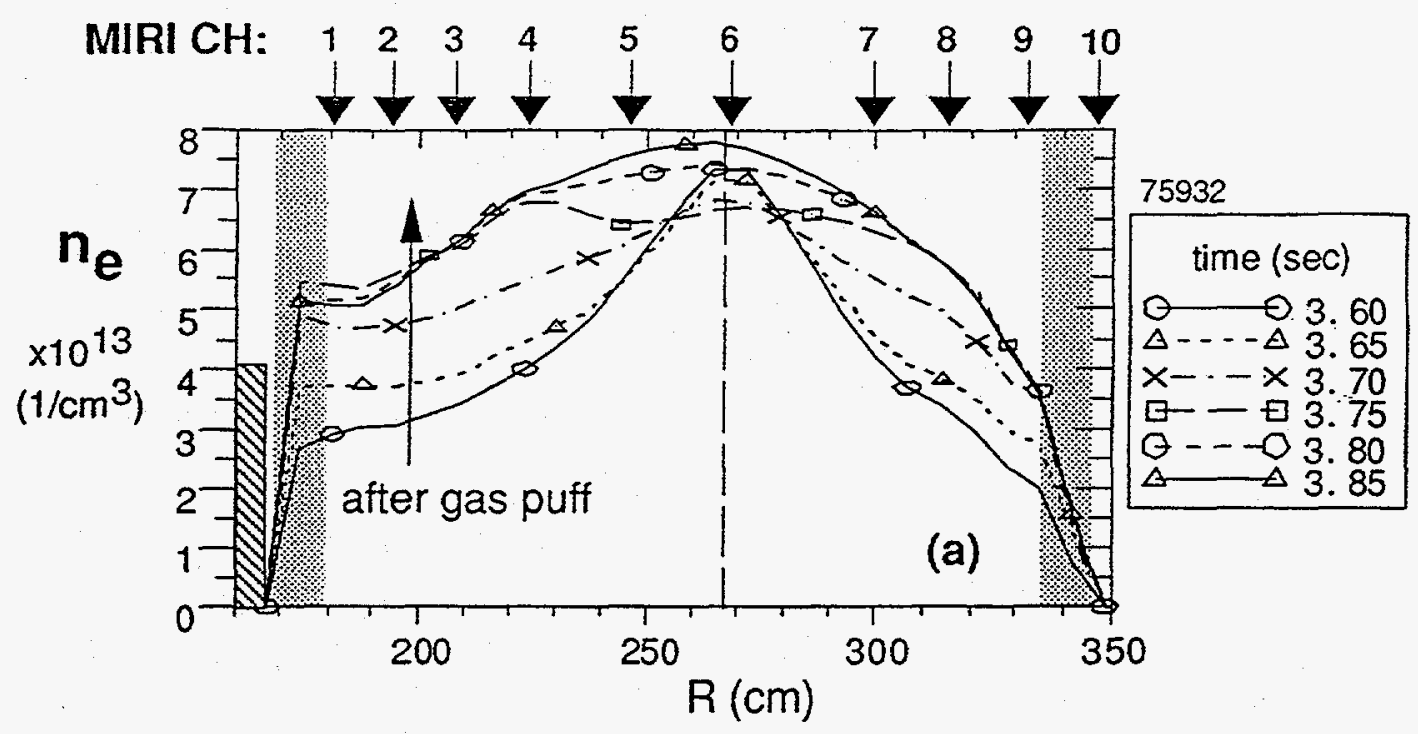

(b)

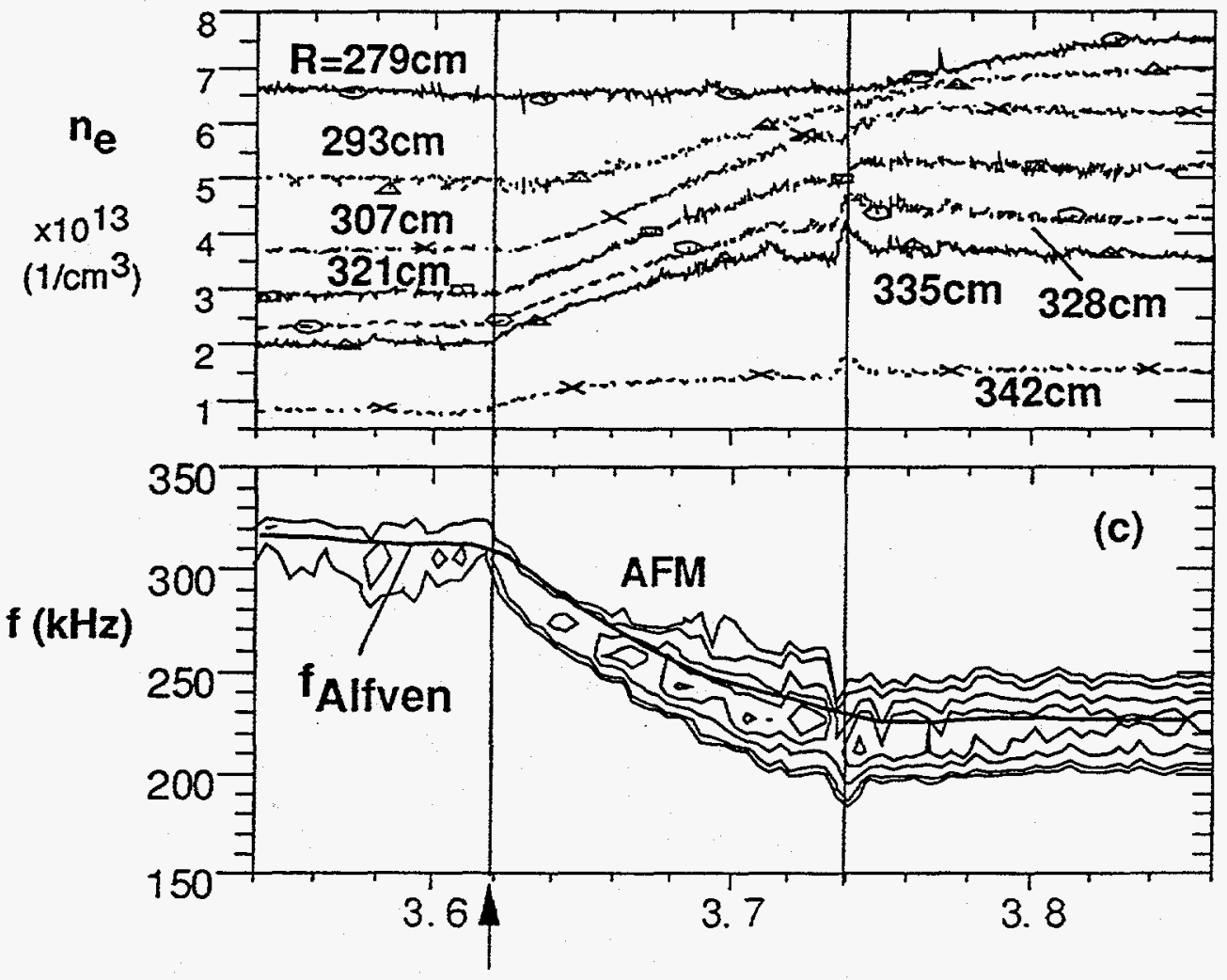

Gas puff time (sec)

Fig. 4 


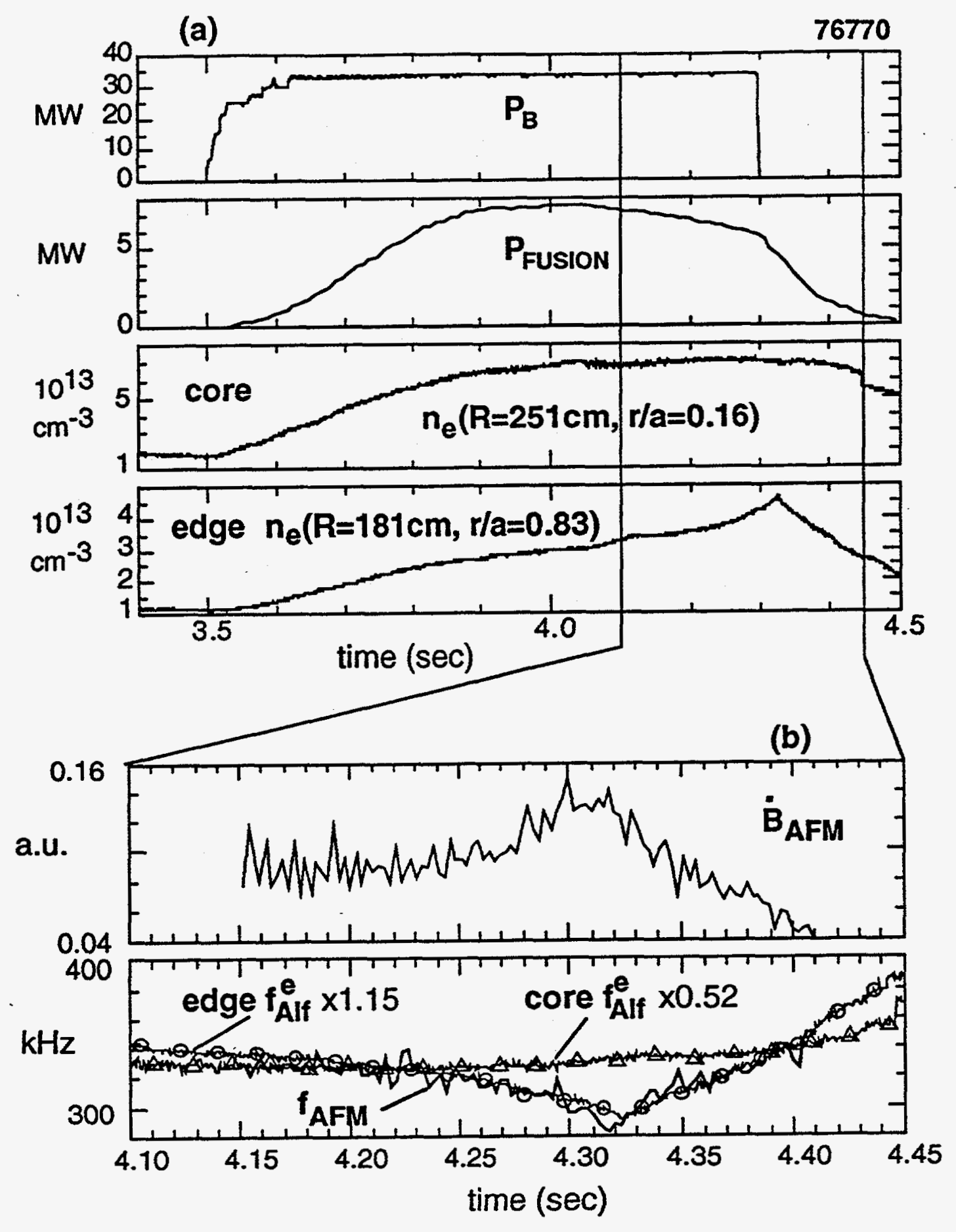

Fig. 5 

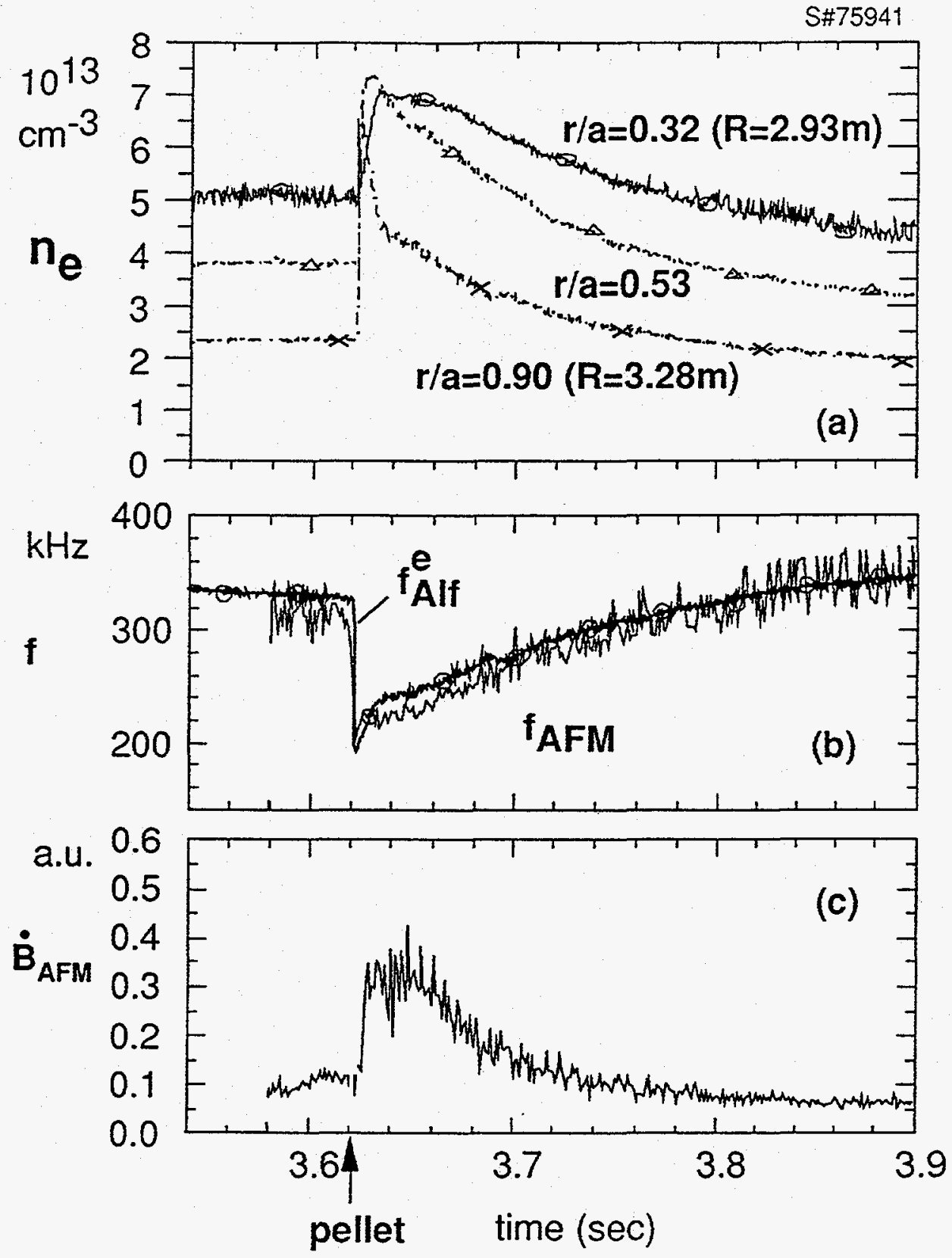

Fig. 6 




(a)

(b)

(c)

(d)

(e)

(f)

(g)

(h)

Fig. 7 


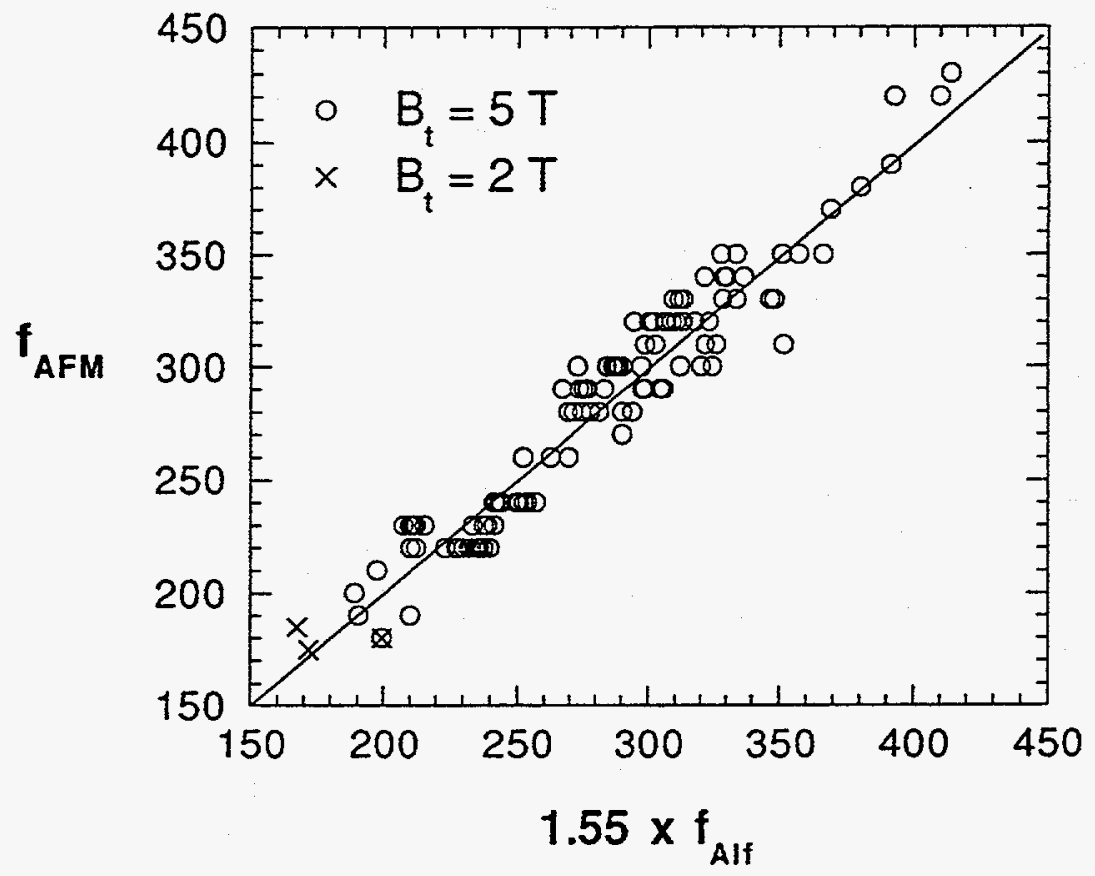

Fig. 8 
(a)

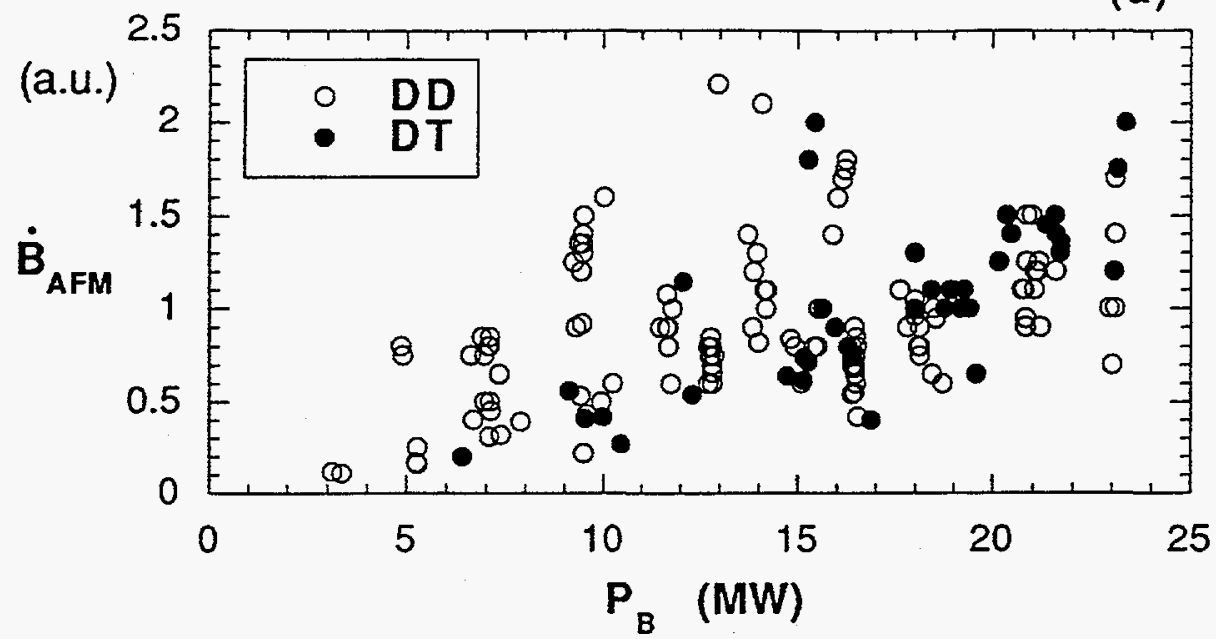

(b)

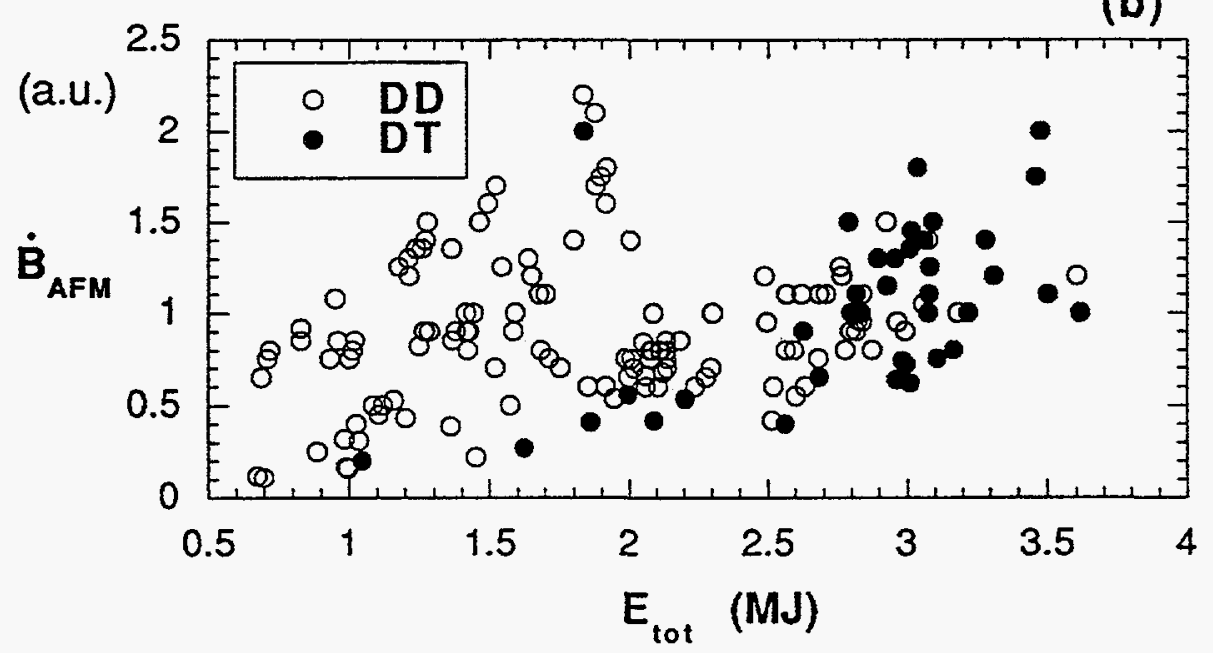

(c)

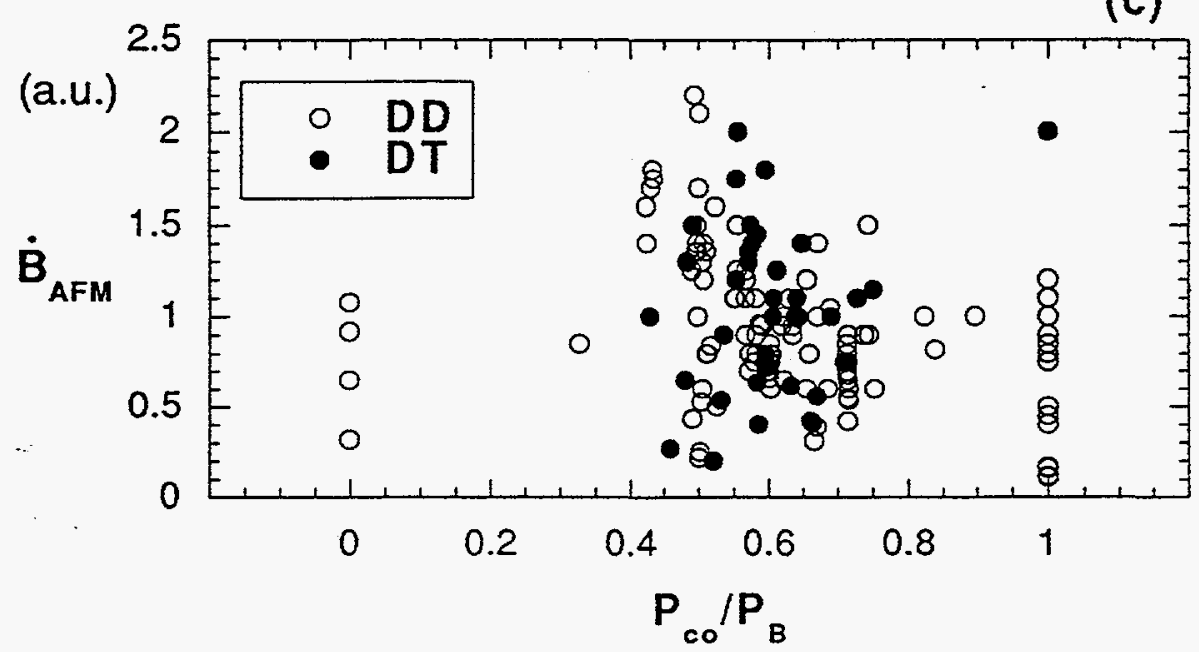

Fig. 9 

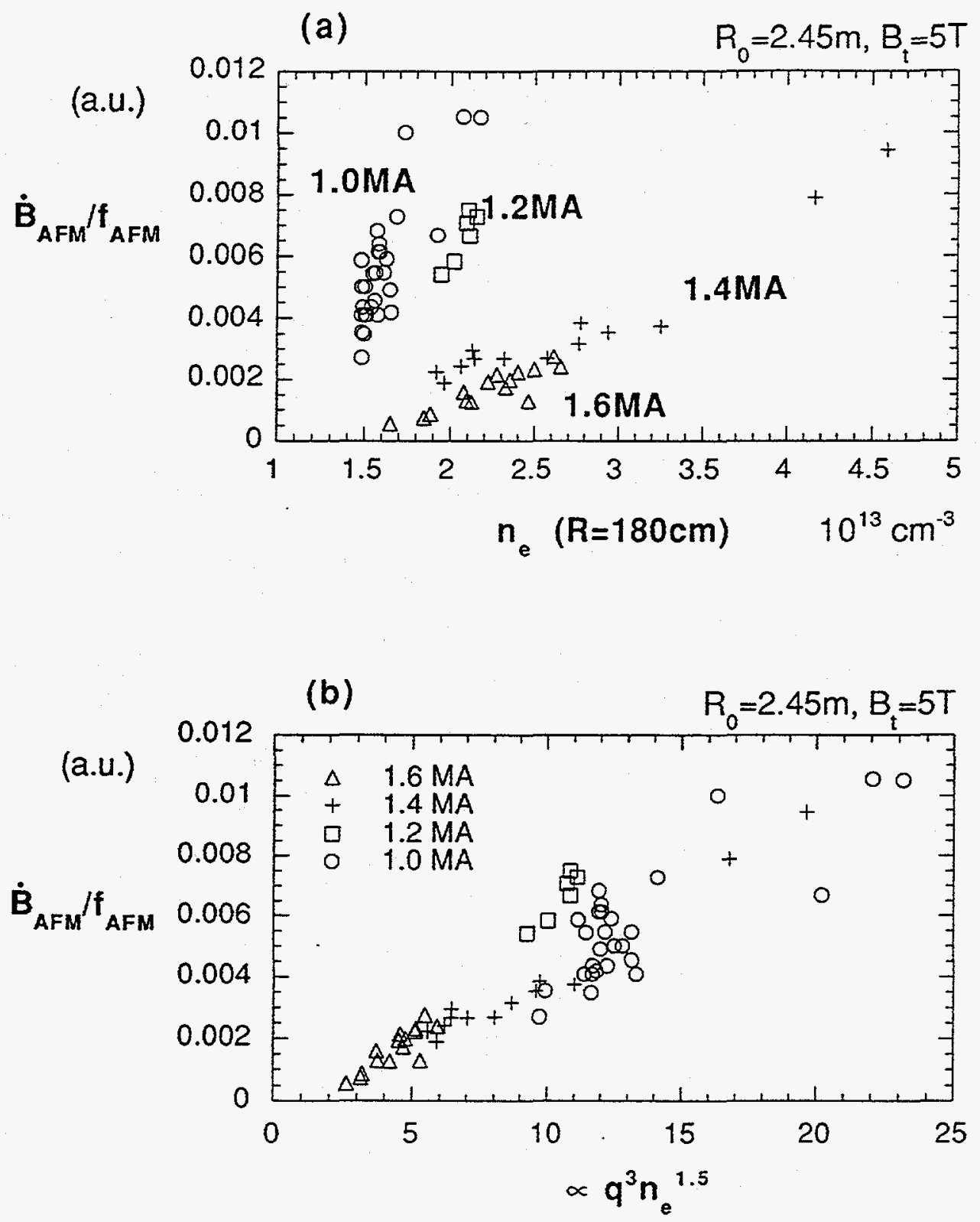

Fig. 10 


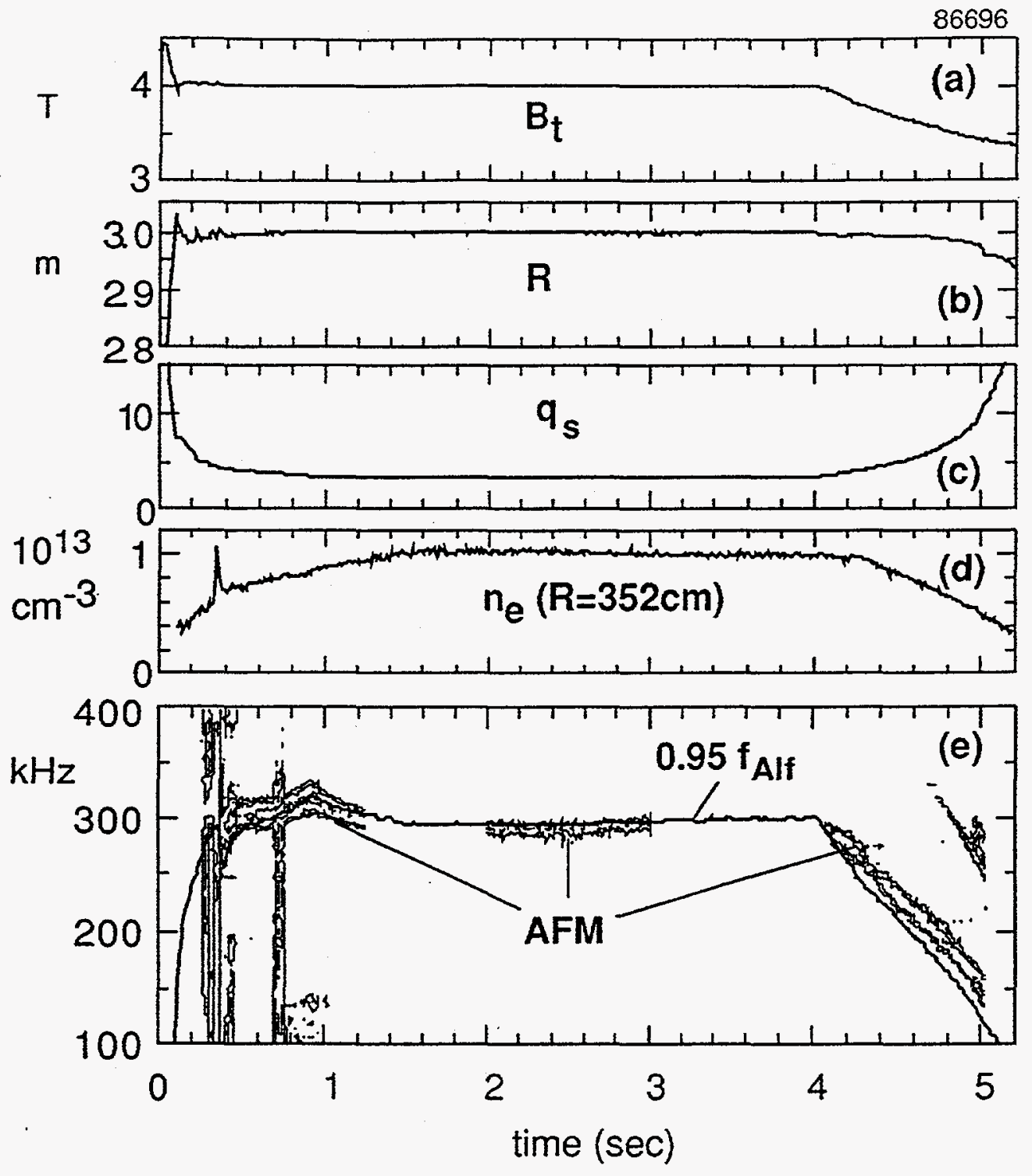

Fig. 11 


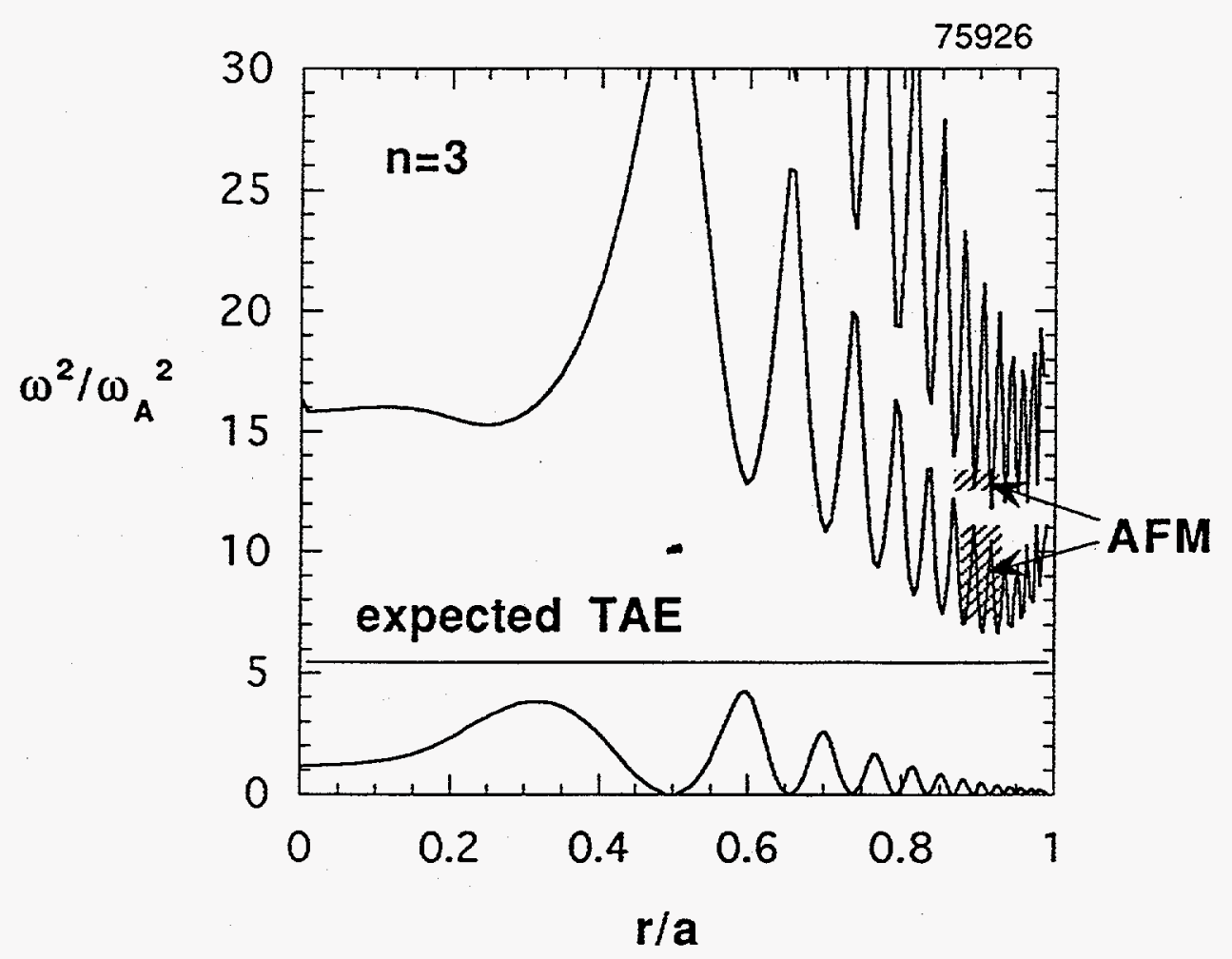

Fig. 12 
Dr. F. Paoloni, Univ. of Wollongong, AUSTRALIA

Prof. R.C. Cross, Univ. of Sydney, AUSTRALIA

Plasma Research Lab., Australian Nat. Univ., AUSTRALIA

Prof. I.R. Jones, Flinders Univ, AUSTRALIA

Prof. F. Cap, inst. for Theoretical Physics, AUSTRIA

Prof. M. Heindler, Institut für Theoretische Physik, AUSTRIA

Prof. M. Goossens, Astronomisch Instituut, BELGIUM

Ecole Royale Militaire, Lab. de Phy. Plasmas, BELGIUM

Commission-European, DG. XII-Fusion Prog., BELGIUM

Prof. R. Boucique, Rijksuniversiteit Gent, BELGIUM

Dr. P.H. Sakanaka, Instituto Fisica, BRAZIL

Prof. Dr. I.C. Nascimento, Instituto Fisica, Sao Paulo, BRAZIL Instituto Nacional De Pesquisas Espaciais-INPE, BRAZIL

Documents Office, Atomic Energy of Canada Ltd., CANADA

Ms. M. Morin, CCFMTokamak de Varennes, CANADA

Dr. M.P. Bachynski, MPB Technologies, Inc., CANADA

Dr. H.M. Skarsgard, Univ. of Saskatchewan, CANADA

Prof. J. Teichmann, Univ. of Montreal, CANADA

Prof. S.R. Sreenivasan, Univ. of Calgary, CANADA

Prof. R. Marchand, INRS-Energie et Materiaux, CANADA

Dr. R. Boiton, Centre canadien de fusion magnétique, CANADA

Dr. C.R. James, Univ. of Alberta, CANADA

Dr. P. Lukác, Komenského Universzita, CZECHO-SLOVAKIA

The Librarian, Culham Laboratory, ENGLAND

Library, R61, Rutherford Appleton Laboratory, ENGLAND

Mrs. S.A. Hutchinson, JET Library, ENGLAND

Dr. S.C. Sharma, Univ. of South Pacific, FIJI ISLANDS

P. Mähönen, Univ. of Helsinki, FINLAND

Prof. M.N. Bussac, Ecole Polytechnique, FRANCE

C. Mouttet, Lab. de Physique des Milieux lonisés, FRANCE

J. Radet, CEN/CADARACHE - Bat 506, FRANCE

Prof. E. Economou, Univ. of Crete, GREECE

Ms. C. Rinni, Univ. of loannina, GREECE

Preprint Library, Hungarian Academy of Sai., HUNGARY

Dr. B. DasGupta, Saha inst of Nuclear Physics, INDIA

Dr. P. Kaw, Inst. for Plasma Research, INDIA

Dr. P. Rosenau, Israel Inst. of Technology, ISRAEL

Librarian, International Center for Theo Physics, ITALY

Miss C. De Palo, Associazione EURATOM-ENEA , ITALY

Dr. G. Grosso, Istituto of Fisica del Plasma, ITALY

Prof. G. Rostangni, Istituto Gas lonizzati Del Cnr, ITALY
Dr. H. Yamato, Toshiba Res \& Devel Center, JAPAN

- Prof. I. Kawakami, Hiroshima Univ., JAPAN

Prof. K. Nishikawa, Hiroshima Univ., JAPAN

Librarian, Naka Fusion Research Establishment, JAERI, JAPAN

Director, Japan Atomic Energy Research Inst., JAPAN

Prof. S. Itoh, Kyushu Univ., JAPAN

Research Info. Ctr., National Instit. for Fusion Science, JAPAN

Prof. S. Tanaka, Kyoto Univ., JAPAN

Library, Kyoto Univ., JAPAN

Prof. N. Inoue, Univ. of Tokyo, JAPAN

Secretary, Plasma Section, Electrotechnical Lab., JAPAN

Dr. O. Mitarai, Kumamoto Inst. of Technology, JAPAN

Dr. G.S. Lee, Korea Basic Sci. Ctr., KOREA

J. Hyeon-Sook, Korea Atomic Energy Research Inst., KOREA

D.1. Choi, The Korea Adv. Inst. of Sci. \& Tect., KOREA

Leandro Melendez Lugo, Inst. Nac1. de Inves. Nucl, MEXICO

Prof. B.S. Liley, Univ, of Waikato, NEW ZEALAND

Inst of Physics, Chinese Acad Sci PEOPLE'S REP. OF CHINA

Library, Inst. of Plasma Physics, PEOPLE'S REP. OF CHINA

Tsinghua Univ. Library, PEOPLE'S REPUBLIC OF CHINA

Z. Li, S.W. Inst Physics, PEOPLE'S REPUBLIC OF CHINA

Prof. J.A.C. Cabral, Instituto Superior Tecnico, PORTUGAL

Prof. M.A. Hellberg, Univ. of Natal, S. AFRICA

Prof. D.E. Kim, Pohang Inst. of Sci. \& Tech., SO. KOREA

Prof. C.I.E.M.A.T, Fusion Division Library, SPAIN

Dr. L. Stenflo, Univ. of UMEA, SWEDEN

Library, Royal inst. of Technology, SWEDEN

Prof. H. Wilhelmson, Chalmers Univ. of Tech., SWEDEN

Centre Phys. Des Plasmas, Ecole Polytech, SWITZERLAND

Bibliotheek, Inst. Voor Plasma-Fysica, THE NETHERLANDS

Asst. Prof. Dr. S. Cakir, Middle East Tech. Univ., TURKEY

Dr. V.A. Glukhikh,Sci. Res. Inst. Electrophys.I Apparatus, USSR

Dr. D.D. Ryutov, Siberian Branch of Academy of Sci., USSR

Dr. G.A. Eliseev, I.V. Kurchatov Inst., USSR

Librarian, The Ukr.SSR Academy of Sciences, USSR

Dr. L.M. Kovrizhnykh, Inst. of General Physics, USSR

Kemforschungsanlage GmbH, Zentralbibliothek, W. GERMANY

Bibliothek, Inst. Für Plasmaforschung, W. GERMANY

Prof. K. Schindler, Ruhr-Universitát Bochum, W. GERMANY

Dr. F. Wagner, (ASDEX), Max-Planck-Institut, W. GERMANY

Librarian, Max-Planck-Institut, W. GERMANY 